\title{
Influence of Brain Atrophy using Semi-quantitative Analysis in [123I ]FP-CIT Single Photon Emission Computed Tomography: A Monte Carlo Simulation Study
}

\section{Hiroki Nosaka}

Clinical Imaging Centrer for Healthcare, Nippon Medical School https://orcid.org/0000-0003-3271-

4238

Masahisa Onoguchi ( $\sim$ onoguchi@staff.kanazawa-u.ac.jp)

Graduate School of Medical Sciences, Kanazawa University

\section{Masaya Suda}

Department of Radiology, Nippon Medical School

\section{Hiroyuki Tsushima}

Graduate of Radiology, Ibaraki Prefectural University of Health Sciences

\section{Satoshi Kurata}

Department of Radiological Technology, Ibaraki Prefectural Central Hospital

\section{Ayano Onoma}

Department of Radiology, Yokohama City University Hospital

\section{Ryosuke Murakawa}

Ibaraki Prefectural University of Health Sciences

\section{Original research}

Keywords: [123I ]FP-CIT, Single photon emission computed tomography, Monte Carlo simulation

Posted Date: January 13th, 2021

DOl: https://doi.org/10.21203/rs.3.rs-140328/v1

License: (9) This work is licensed under a Creative Commons Attribution 4.0 International License. Read Full License 


\section{Abstract}

Background The specific binding ratio (SBR), an objective indicator of $\mathrm{N}$ - $\omega$-fluoropropyl-2 $\beta$-carbomethoxy$3 \beta-(4-[123 \mid]$ iodophenyl) nortropane ([123]]FP-CIT) single photon emission computed tomography (SPECT) could be used for the diagnosis of Parkinson' disease and dementia with Lewy bodies. One of the problems of SBR analysis is the setting position of the volume of interest (VOI) might contain cerebral ventricles and cerebral groove. These areas become prominent in analysis with brain atrophy, however these studies have not been done sufficiently.

Results We examined the influence of brain atrophy in SBR analysis using Monte Carlo simulation. The brain atrophy model (BAM) which set the simulate of brain atrophy to 5 stages was prepared using morphological operation. Projection data was created based on BAM, and SPECT reconstruction was carried out. The counts of striatal VOI decreased as brain atrophy progresses. Similarly, the counts of the reference VOI also decreased as brain atrophy progressed. In the striatal VOI counts and the reference VOI counts, the reference VOI counts was more affected by brain atrophy than striatal VOI counts. The SBR calculated from these values was overestimated as brain atrophy progresses.

Conclusions It was suggested that the SBR could be overestimated in the secular change of the case in which brain atrophy advanced.

\section{Background}

It has been known for over three decades that the striatal dopamine transporter (DAT) density decreases in dopaminergic degenerative disorders such as Parkinson's disease (PD) and dementia with Lewy bodies (DLB) $[1,2]$. Single photon emission computed tomography (SPECT) imaging of the DAT density with N-

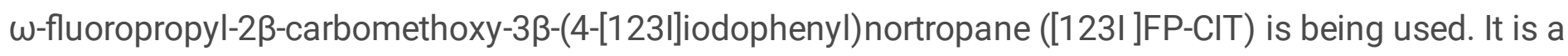
recently developed imaging tool that could prove extremely useful for the diagnosis of these diseases group [3-5].

To evaluate DAT density decline with [123I ]FP-CIT SPECT, semiquantitative analysis using specific binding ratio (SBR) is widely employed [6, 7]. Proposed by Bolt in 2006, the SBR is one of the most established among a plethora of analytical strategies in this field [8]. SBR is computed as the ratio of specific binding counts from the striatal volume of interest (VOI) to nonspecific binding counts from a reference VOI, devoid of DAT. The formula of SBR for this study is shown below (1).

$$
\mathrm{SBR}=\left\{\frac{\mathrm{Cs} \text { total }}{C r}-\mathrm{Vs}_{\mathrm{VOI}}\right\}\left(\frac{1}{V s}\right) \ldots(1)
$$

SBR: specific binding ratio by Bolt

$\mathrm{Cs}_{\text {total }}$ : striatal VOI total counts [counts] 
Cr: mean counts per volume in the reference VOI [counts $/ \mathrm{cm}^{3}$ ]

$\mathrm{Vs}_{\mathrm{VO}}$ : volume of striatal $\mathrm{VOI}\left[\mathrm{cm}^{3}\right]$

Vs: volume of the striatum $\left[\mathrm{cm}^{3}\right]$

The use of a large VOI for striatum is recommended with the purpose of minimizing the influence of the partial volume effect [8]. However, a large striatal VOI may contain several low accumulation areas due to the adjacent cerebral ventricles and sulci, making the SBR sensitive to the volume of these anatomical structures. Enlargement of cerebral ventricles and sulci in brain atrophy patients represents a common issue in diagnostic imaging.

Several studies have independently reported their influence on the SBR calculation using digital phantoms [9-11]. However, the joint influence of cerebral ventricles and sulci has not been previously examined. Therefore, the goal of this study is to investigate how brain atrophy affects SBR using a Monte Carlo simulation.

\section{Methods}

\section{Brain atrophy model}

A brain atrophy model (BAM) was generated from the Zubal brain digital phantom provided by Yale University [12-14]. Since the Zubal head phantom is based on high-resolution MRI images of normal adults, the derived BAM could be reproduced detailed clinical studies.

The BAM was created out of the following four segments extracted from the Zubal phantom. The bone regions were generated from the skull. The striatum regions were putamen and caudate nuclei extracted from the phantom. The cerebral ventricle regions were the third ventricle, fourth ventricle, lateral ventricles, cerebral aqueduct, and spinal cord. As for the brain parenchyma regions, white matter and gray matter were extracted from the phantom. Regions from the cerebral ventricles were also extracted to generate homogeneous brain parenchyma.

Background regions were created by subtracting cerebral ventricle regions from brain parenchyma regions. These background regions were used as reference regions for [123I ]FP-CIT SPECT nonspecific binding counts. In addition, cerebral ventricle and brain parenchyma regions underwent morphological operation to reproduce brain atrophy as described below using the image processing software ImageJ.

Finally, the BAM was created adding together bone regions, striatum regions and background regions. This process is shown in Fig. 1.

To simulate five patterns of brain atrophy, the BAM was modified by 0.5 pixel from -1 to +1 . The ratio of the striatal to brain parenchyma accumulation was set to eight to one. Brain atrophy levels were defined 
in descending order from 1 to 5 , and level 5 corresponding to the most severe damage. The created BAM was shown in Fig. 2.

\section{Monte Carlo simulation}

Projection data were generated from the BAM using the Monte Carlo simulation code SIMIND provided by LUND University [15-20]. This code can output several ${ }^{123}$ I-ioflupane simulations. Simulations were performed assuming the detector structure of Gamma-Camera as the one designed for BrightView XCT(Hitachi, Ltd. Tokyo, Japan). BrightView XCT is provided with a dual Nal crystal detector, optimized for size (field of view: $40.6 \times 53.9 \mathrm{~cm}$; thickness: $9.5 \mathrm{~mm}$ ). A low-energy high-resolution collimator was simulated. And a $7.0 \mathrm{~cm}$ photomultiplier tube was coupled with the Nal detector. ${ }^{123}$ I emissions were simulated as a radiation source.

Projection data were simulated as a $128 \times 128$ pixels matrix, collected using 90 projections by $4^{\circ}$ steps. The sum of all projection data has been adjusted to 1.5 million counts. The pixel size was set to $3.2 \mathrm{~mm}$. The main energy window was kept within the 143.1 to $174.9 \mathrm{keV}$ range, it was $159 \mathrm{keV} \pm 10 \%$. Five sets of data were simulated per condition.

We adopted the Zubal head phantom density specified by SIMIND: the bone regions density was set to $1.22 \mathrm{~g} / \mathrm{cm}^{3}$, while the striatum, cerebral ventricles, and brain parenchyma density was $1.04 \mathrm{~g} / \mathrm{cm}^{3}$.

\section{Image reconstruction}

The simulated projection data were reconstructed using the Prominence Processor software, version 3.1. The reconstruction conditions were based on the Japanese [123I ]FP-CIT clinical diagnostic guidelines [21]. Filtered back projection was performed with a Butterworth filter, with a cutoff frequency of 0.5 cycles/cm and an order of 8 . Attenuation and scatter correction were not performed.

\section{Image analysis}

The reconstructed images were analyzed thanks to the image analysis software DatView(Nihon MediPhysics, Japan). This software allows computing the SBR based on the Bolt's analysis method. For the purpose of this quantification, the images were automatically aligned to the Anterior comisure-Posterior comisure line. Since the set of BAM was based on the same Zubal phantom, the inclination of the brain did not vary within the set. Therefore, the correction for the brain inclination was applied across models. The striatal VOI volume $\left(\mathrm{Vs}_{\mathrm{VOI}}\right)$ and striatal volume $(\mathrm{Vs})$ were $283.12 \mathrm{~cm}^{3}$ and $22.4 \mathrm{~cm}^{3}$, respectively, and the same values were used for all SBR calculations.

The threshold value for the reference VOI was determined based on the $50 \%$ of the highest count value. In order to exclude margins with partial volume effect, the borders of the reference area were set $20 \mathrm{~mm}$ 
inwards from the VOI margins. All the analysis parameters were selected according to Bolt's protocol.

SBR quantification was performed on all simulated images for each of the right and left striatal VOI, and five independent counts were averaged per condition. The average value across the right and left side of the brain was taken as the output measure.

Significant difference tests were performed for each value using the Student's $t$ test and a statistical significance was considered at $p<0.05$.

\section{Results}

\section{BAM}

Table 1 shows the volume of the BAM background regions created by morphological operation. As the brain atrophy progresses, the volume of the background regions decreases. Atrophy level 3 corresponds to the original Zubal head phantom with no morphological operation applied.

Table 1

Changes in the brain atrophy level and its volume

\begin{tabular}{|lll|}
\hline atrophy level & morphological operation & BG regions $\left[\mathrm{cm}^{3}\right]$ \\
\hline level 1 & +1.0 pixel & 1519.34 \\
\hline level 2 & +0.5 pixel & 1484.37 \\
\hline level 3 & No processing & 1387.41 \\
\hline level 4 & -0.5 pixel & 1285.63 \\
\hline level 5 & -1.0 pixel & 1243.29 \\
\hline $\begin{array}{l}\text { The background (BG) regions were changed based on level 3. The higher the level, the greater the } \\
\text { brain atrophy. }\end{array}$ \\
\hline
\end{tabular}

\section{Striatal and reference VOI value}

Table 2 shows the mean counts per volume for the striatal VOI (Cs) and the reference $\mathrm{VOI}(\mathrm{Cr})$ and respective percent change for atrophy level 3 . The $\mathrm{Cr}$ and the striatal $\mathrm{VOI}$ total counts ( $\left.\mathrm{Cs}_{\text {total }}\right)$ obtained by multiplying Cs by the number of volume of the striatal VOI were used for SBR computation. 
Table 2

Changes in mean counts in the striatal VOI and the reference VOI

\begin{tabular}{|lllll|}
\hline atrophy level & \multicolumn{3}{l}{ Striatal VOI } & \multicolumn{3}{l|}{ Reference VOI } \\
\cline { 2 - 5 } & mean counts (Cs) & percent change [\%] & mean counts (Cr) & percent change [\%] \\
\hline level 1 & $35.32 \pm 0.16$ & 3.64 & $25.53 \pm 0.22$ & 4.93 \\
\hline level 2 & $35.14 \pm 0.11$ & 3.11 & $25.14 \pm 0.19$ & 3.33 \\
\hline level 3 & $34.08 \pm 0.05$ & 0.00 & $24.33 \pm 0.11$ & 0.00 \\
\hline level 4 & $33.07 \pm 0.13$ & -2.96 & $23.45 \pm 0.18$ & -3.62 \\
\hline level 5 & $32.40 \pm 0.16$ & -4.93 & $22.93 \pm 0.28$ & -5.75 \\
\hline $\begin{array}{l}\text { The striatal Vol mean counts were lower at higher atrophy levels. The reference VOI mean counts } \\
\text { showed the same tendency. }\end{array}$ & & \\
\hline
\end{tabular}

Cs and $\mathrm{Cr}$ decreased along with the increase of brain atrophy level and reduction of the BAM background regions. The percent change of $\mathrm{Cr}$ was larger than the percent change of $\mathrm{Cs}$ for the same atrophy level, implying that $\mathrm{Cr}$ was more influenced by the brain atrophy than $\mathrm{Cs}$. In all comparisons except atrophy level1 vs level2, the $C$ s values has a significant difference $(p<0.05)$. In reference $\mathrm{VOI}$, a significant difference was observed in all $\mathrm{Cr}$ values $(\mathrm{p}<0.05)$.

The relationship between $\mathrm{Cs}$ and the volume of the BAM background regions is displayed in Fig. 3 . Similarly, the relationship between $\mathrm{Cr}$ and the volume of the BAM background regions is displayed in Fig. 4. Figure 3 and Fig. 4 shows that both $\mathrm{Cs}$ and $\mathrm{Cr}$ has a strong positive correlation with the volume of the BAM background regions.

\section{SBR}

Table 3 shows the SBR measurements for each atrophy level and the percent change for atrophy level 3 . The SBR was overestimated as the volume of the atrophy level increased. 
Table 3

Relationship between the brain atrophy leval and the SBR

\begin{tabular}{|lll|}
\hline atrophy level & SBR & percent change [\%] \\
\hline level 1 & $4.85 \pm 0.21$ & -4.34 \\
\hline level 2 & $5.03 \pm 0.19$ & -0.79 \\
\hline level 3 & $5.07 \pm 0.10$ & 0.00 \\
\hline level 4 & $5.19 \pm 019$ & 2.37 \\
\hline level 5 & $5.23 \pm 0.24$ & 3.16 \\
\hline $\begin{array}{l}\text { The higher the atrophy level, the higher the SBR. In other words, SBR was overestimated compared to } \\
\text { when there is no atrophy. }\end{array}$ \\
\hline
\end{tabular}

A significant difference was observed in the comparison between atrophy level 1 to atrophy level 4 and 5 . There was no significant difference in the comparison of other values.

The relationship between SBR and the volume of the BAM background regions is displayed in Fig. 5 . These values were inversely correlated. In other words, SBR was overestimated with increasing brain atrophy and decreasing BAM background regions.

\section{Discussion}

This study was investigated how brain atrophy affects the SBR by relying on the Monte Carlo simulation method. Different degrees of brain atrophy severity were modeled by morphological operation to vary the volumes of brain parenchyma and cerebral ventricles. Using this method, it was possible to determine the volume of the brain in detail. By knowing the volume of the brain, we succeeded in investigating the relationship between brain atrophy and SBR.

The striatal VOI mean binding count was significantly correlated with the volume of the BAM background regions (Table 2 and Fig. 3). Specifically, this measured value decreased as the volume of the brain parenchyma regions decreased due to brain atrophy. Similarly, the reference VOI mean binding count showed a significant correlation with the brain parenchyma regions (Table 2 and Fig. 4). As the volume of the brain parenchyma regions decreased, the counts of the reference VOI decreased. These phenomena are usually attributed to the relative increase in cerebral sulci and ventricles regions volume in each VOI due to brain atrophy. To minimize the influence of the partial volume effect, the large striatal VOI is used for calculation of SBR in the Bolt method. Therefore, in the case with brain atrophy, it is possible that regions where [123I ]FP-CIT dose not accumulate such as cerebral sulci and ventricles is included within striatal VOI. It is considered that such contamination of regions where [123I ]FP-CIT does not accumulate cause decreased counts in striatal VOI.

The reference VOI was more strongly affected by brain atrophy as compared to the striatal VOI. It could be explained with Bolt's analysis procedure [8]. First, when setting the reference VOI, smoothing 
processing is performed three times within the reference regions and therefore excluding the striatal VOI. In addition, $50 \%$ of the highest count of the reference VOI is set as a threshold value. Finally, the area $20 \mathrm{~mm}$ inwards from the margins is set as the reference VOI. The advantage of this method is that it takes into account the partial volume effect by excluding the marginal areas of the brain. Therefore, in case of brain atrophy, the affected areas such as cerebral sulci could be excluded. However, the central part of the brain has different consequences. The volumes of the central sulcus and Sylvian fissure also change in brain atrophy. Even if the marginal areas of the brain were excluded, many of these nonintegrated areas were not. Consequently, the reference VOI was more strongly affected than the striatal VOI.

The change in the SBR value could be explained from the SBR calculation formula. In the SBR computation, the striatal $\mathrm{VOI}\left(\mathrm{Vs}_{\mathrm{VO}}\right)$ volume and the striatum $(\mathrm{Vs})$ volume were kept constant across simulations. $V s_{V O I}$ and $V s$ were $283.12 \mathrm{~cm}^{3}$ and $22.4 \mathrm{~cm}^{3}$. Therefore, the $V s_{V O I}$ and $V s$ do not affect the SBR analysis. As a result, only the counts of the striatal VOI total counts $\left(\mathrm{Cs}_{\mathrm{total}}\right)$ and the mean counts per volume in the reference $\mathrm{VOI}(\mathrm{Cr})$ affected the output value in formula 1 . That is, the ratio $\mathrm{Cs}_{\text {total }} / \mathrm{Cr}$ became the crucial parameter for the SBR calculation. From Table 2, the percent change in the reference VOI was larger than the one in the striatal VOI. Therefore, the SBR likely reflects the change of $\mathrm{Cr}$, being inversely related to $\mathrm{Cr}$. Consequently, the SBR was overestimated in the model of cerebral atrophy in which the value of $\mathrm{Cr}$ more decreased.

As evident from Table 3 and Fig. 5, the SBR calculated using the Bolt's approach showed a significant negative correlation with brain atrophy. In other words, the SBR was overestimated as the brain parenchyma volume decreased as a consequence of brain atrophy. Our findings are in accordance with the observations made by Furuta et al., who only altered the size of the brain ventricles [10].

In this study, brain atrophy particularly affected the reference VOI. Several methods for calculating the SBR rely on a reference VOI corresponding to the whole brain or the occipital lobe. In the SBR analysis of patients with brain atrophy, it should be considered a preferable option to modify the position of the reference VOI depending on of degree of regional brain atrophy. However, Watanabe's report highlighted that the SBR values change with the setting of the reference VOI threshold [11]. Therefore it is risky to shift the setting range of the threshold value and VOI location unnecessarily, and further investigations are needed to ascertain the pros and cons of this option. Alternatively, it might be necessary to introduce a new analysis method independent of a reference VOI. Possible alternatives are estimates only based on the VOI of the striatum or on the accumulation shape of the striatum.

Many patients undergoing [123I ]FP-CIT SPECT examination are elderly. It has been reported that agerelated causes brain atrophy[22, 23]. We showed that brain atrophy induced an overestimation of SBR values. This overestimation is a confounding factor for the estimation of the DAT density decrease when the diagnosis of dopaminergic degenerative disorders. 
Overestimation of SBR may be a particular problem in diseases in which the whole striatum accumulation decreases such as DLB. It has been shown that SBR estimates decrease in DLB with the time course of the [123I ]FP-CIT SPECT investigation. Recent data analysis research of ENC-DAT shows that SBR decreases with age $[24,25]$. However, in cases with advanced brain atrophy due to aging, this effect was counteracted by the related overestimation of the SBR.

In this study, the accumulation of the striatum was constant. Therefore, it is necessary to consider alternative unbiased models to properly measure the decreased striatal [123I ]FP-CIT SPECT accumulation in diseases such as PD and DLB.

\section{Conclusions}

This Monte Carlo simulation study clarified that SBR values are overestimated in patients with brain atrophy. Therefore, the influence of brain atrophy should be taken into account in the SBR computation.

\section{Abbreviations}

$\mathrm{SBR}=$ specific binding ratio

SPECT $=$ single photon emission computed tomography

$\mathrm{VOI}=$ volume of interest

$\mathrm{BAM}=$ brain atrophy model

DAT $=$ dopamine transporter

PD = Parkinson's disease

DLB = dementia with Lewy bodies

$\mathrm{Vs}_{\mathrm{VOI}}=$ striatal $\mathrm{VOI}$ volume

Vs $=$ striatal volume

$\mathrm{Cs}=$ mean counts per volume for striatal VOI

$\mathrm{Cr}=$ mean counts per volume for reference $\mathrm{VOI}$

$\mathrm{Cs}_{\text {total }}=$ striatal $\mathrm{VOI}$ total counts

\section{Declarations}

Ethics approval and consent to participate 


\section{Consent for publication}

Not applicable

\section{Availability of data and material}

Not applicable

\section{Competing interests}

Not applicable

\section{Funding}

Not applicable

\section{Authors' contributions}

MO set guidelines for this study, reviewed the analytical values, and was deeply involved in this treatise. MS and AO contributed to the treatise by presenting clinical problems and their solutions. HT made a great contribution to the manuscript by proposing the optimal analysis method. SK and RM helped with the simulation analysis and contributed to the analysis. All authors read and approved the final manuscript.

\section{Acknowledgements}

Not applicable

\section{Authors' information (optional)}

Not applicable

\section{References}

1. Takano K, MATSUMURA K, Watanabe Y, Yamada T, Kubo H, Naitou Y, Narita Y, Kuzuhara S, Takeda K. Phase 1 Clinical Study of ${ }^{123}$ I-FP-CIT, a New Radioligand for Evaluating Dopamine Transporter by 
SPECT (II) : Tracer Kinetics in the Brain囚JSNM 1999ه36ه1013-1022.

2. Piggott MA, Perry EK, Marshall EF, McKeith IG, Johnson M, Melrose HL, Court JA, Lloyd S, Fairbairn A, Brown A, Thompson P, Perry RH. Nigrostriatal Dopaminergic Activities in Dementia with Lewy Bodies in Relation to Neuroleptic Sensitivity: Comparisons with Parkinson's Disease. Biol Psychiatry. 1998;44:765-774.

3. McKeith IG, Dickson DW, Lowe J, Emre M, O'Brien JT, Feldman H, Cummings J, Duda JE, Lippa C, Perry EK, Aarsland D, Arai H, Ballard CG, Boeve B, Burn DJ, Costa D, Del Ser T, Dubois B, Galasko D, Gauthier S, Goetz CG, Gomez-Tortosa E, Halliday G, Hansen LA, Hardy J, Iwatsubo T, Kalaria RN, Kaufer D, Kenny RA, Korczyn A, Kosaka K, Lee VM, Lees A, Litvan I, Londos E, Lopez OL, Minoshima S, Mizuno Y, Molina JA, Mukaetova-Ladinska EB, Pasquier F, Perry RH, Schulz JB, Trojanowski JQ, Yamada M. Consortium on DLB囚Diagnosis and management of dementia with Lewy bodies : Third report of DLB consortium. Neurology. 2005;65(12):1863-1872.

4. Niznik HB, Fogel EF, Fassos FF, Seeman P. The Dopamine Transporter is absent in parkinsonian putamen and reduced in the caudate nucleus. J Neurochem. 1991;56(1):192-198.

5. McKeith I, O'Brien J, Walker Z, Tatsch K『Booij J, Darcourt J, Padovani A, Giubbini R, Bonuccelli U, Volterrani D, Holmes C, Kemp P, Tabet N, Meyer I, Reininger C;DLB Study Group. Sensitivity and specificity of dopamine transporter imaging with ${ }^{123}$-FP-CIT SPECT in dementia with Lewy bodies ; A

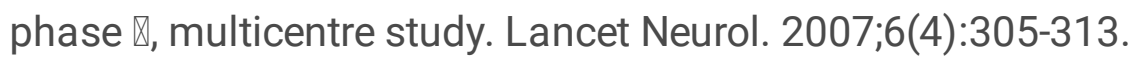

6. Jan Booij, Jan B.A. Habraken, Paul Bergmans, Gerrit Tissingh, Ania Winogrodzka, Erik Ch.Wolters, Anton G.M. Janssen, Johannes C. Stoof, Eric A. van Royen. Imaging of Dopamine Transporters with lodine-123FP-CIT SPECT in Healthy Controls and Patients with Parkinson's Disease. J Nucl Med. 1998; 39:1879-1884.

7. K Badiavas, E Molyvda, K Psarrakos, I lakovou, N Karayzas, M Tsolaki. SPECT imaging evaluation in movement disorders: far beyond visual assessment. Eur J Nucl Med Mol Imaging. 2011.;38:764773.

8. Tossici-Bolt L, Hoffmann SM, Kemp PM, Mehta RL, Fleming JS. Quantification of [ ${ }^{123}$ ] $]$ FP-CIT SPECT brain images: an accurate technique for measurement of the specific binding ratio. Eur $\mathrm{J}$ Nucl Med Mol Imaging. 2006;33(12):1491-1499.

9. Washimi M, Yamaki N, Yanagisawa M. A Study on the Effect by Ventricles on Quantitative Index in 123/-Ioflupane SPECT Images. JSNMT 2016;36(4):510-515》

10. Furuta A, Onishi H, Nakamoto K. Development of Realistic Striatal Digital Brain (SDB) Phantom for ${ }^{123}$ I-FP-CIT SPECT and Effect on Ventricle in the Brain for Semi-quantitative Index of Specific Binding Ratio.JSRT 2017;73(10):1018-1027.

11. Watanabe A, Inoue Y, Asano Y, Kikuchi K, Miyatake H, Tokusige T. Examination of a Method to Determine the Reference Region for Calculating the Specific Binding Ratio in Dopamine Transporter Imaging. JSRT 2017;73(4):291-297. 
12. Zubal IG, Harrell CR. Voxel Based Monte Carlo Calculations of Nuclear Medicine Images and Applied Variance Reduction Techniques. Image and Vision Computing. 1992;10(6):342-348.

13. Zubal IG, Harrell CR, Esser PD. Monte Carlo determination of emerging energy spectra for diagnostically realistic radiopharmaceutical distributions. Nuclear Instruments and Methods in Physics Research Section A. 1990;299:544-547.

14. Zubal IG, Harrell CR, Smith EO囚Rattner Z, Gindi G, Hoffer PB. Computerized three-dimensional segmented human anatomy. Medical Physics. 1994;21(2):299-302,1994.

15. Ljungberg M, Strand SE. A Monte Carlo program for the simulation of scintillation camera characteristics. Comput Methods Programs Biomed. 1989;29(4):257-272】

16. Bahreyni Toossi MT, Islamian JP, Momennezhad M, Ljungberg M, Naseri SH. SIMIND Monte Carlo simulation of a single photon emission CT. J Med Phys. 2010;35(1):42-47.

17. Buvat I, Cactiglioni I. Monte Carlo simulations in SPET and PET. Q J Nucl Med. 2002;46(1):48-61.

18. The Japanese Society of Radiolodical Technology's sub group Nuclear Medicine Section. ABC book of experiment in nuclear medicine for beginners. JSRT;2016. p.94-111.

19. Shirakawa S, Ushiroda T, Hashimoto H, Tadokoro M, Uno M, Tsujimoto M, Ishiguro M Toyama $H$. Construction of the Quantitative Analysis Environment Using Monte Carlo Simulation. JSNMT 33区 $367 \varangle 376,2013$

20. Kouch W, Bartenstein P, la Fougère C. Radius dependence of FP-CIT quantification: a Monte Carlobased simulation study. Ann Nucl Med. 2014;28(2):103-111.

21. Japan Society of Nuclear Medicine, The Japanese Council of Nuclear Neuroimaging, editors. Ioflupane Clinical Practice Guidelines Second Edition [Translated from Japanese.]. 2017. p3-18区

22. Ito $M$, Hatazawa J, Yamaura H, Matsuzawa T: Age-related brain atrophy and mental deterioration-A study with computed tomography. Br J Radiol 54: 384-390, 1981.

23. Takeda S, Matsuzawa T: Brain atrophy and mental deterioration. Sci Rep Res Inst Tohoku Univ-C 31: 49-52, 1984

24. Varrone A, Dickson JC, Tossici-Bolt L, Sera T, Asenbaum S, Booij J, et al. European multicentre database of healthy controls for [123I]FP-CIT SPECT (ENC-DAT): age-related effects, gender differences and evaluation of different methods of analysis. Eur J Nucl Med Mol Imaging. 2013;40:213-27

25. Nobili F, Naseri M, De Carli F, Asenbaum S, Booij J, Darcourt J, et al. Automatic semi-quantification of [123I]FP-CIT SPECT scans in healthy volunteers using BasGan version 2: results from the ENC-DAT database. Eur J Nucl Med Mol Imaging. 2013;40:565-73

\section{Figures}




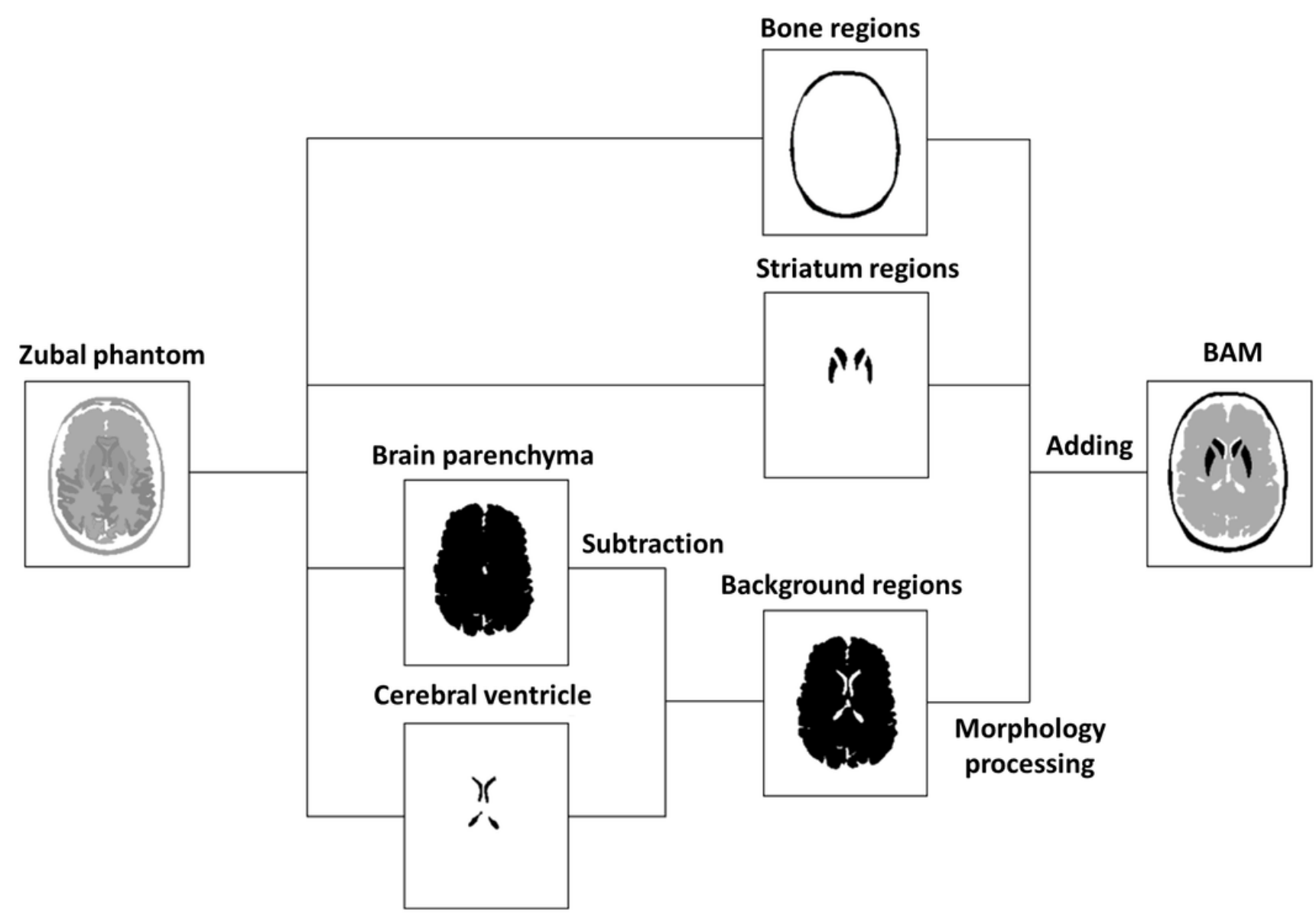

Figure 1

At first, four areas were extracted from the MRI-based Zubal head phantom. Next, background regions were created by differentiating the cerebral ventricle regions from the brain parenchyma regions. Finally, BAM was generated by combining bone regions, striatum regions and background regions subjected to morphological operation.

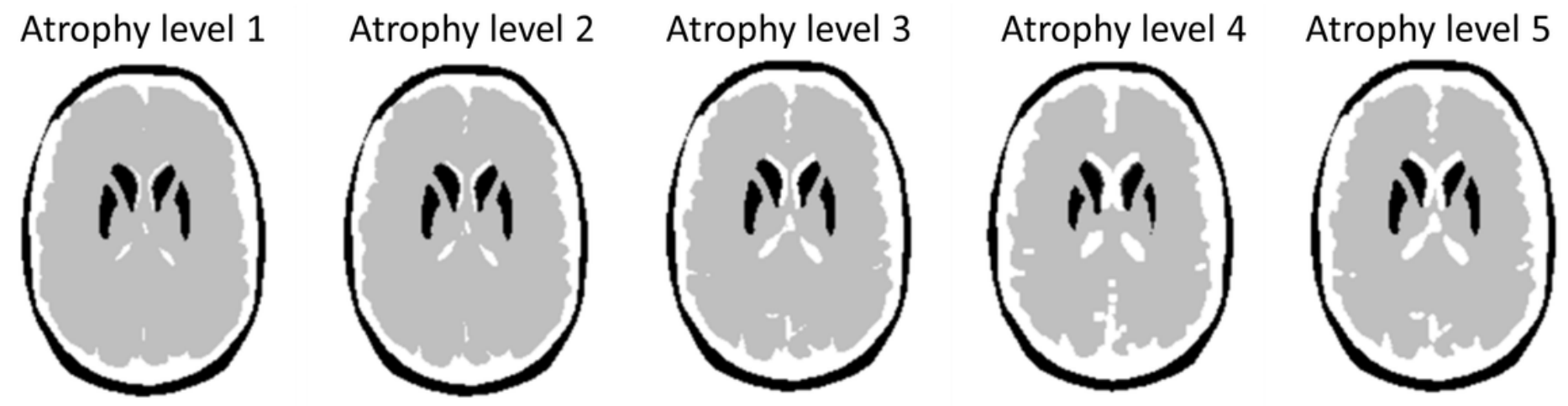

Figure 2

5 patterns of BAM were created. The atrophy level 5 corresponds to the most severe brain damage. The atrophy level 3 corresponds to the original Zubal head phantom with no morphological operation applied. 


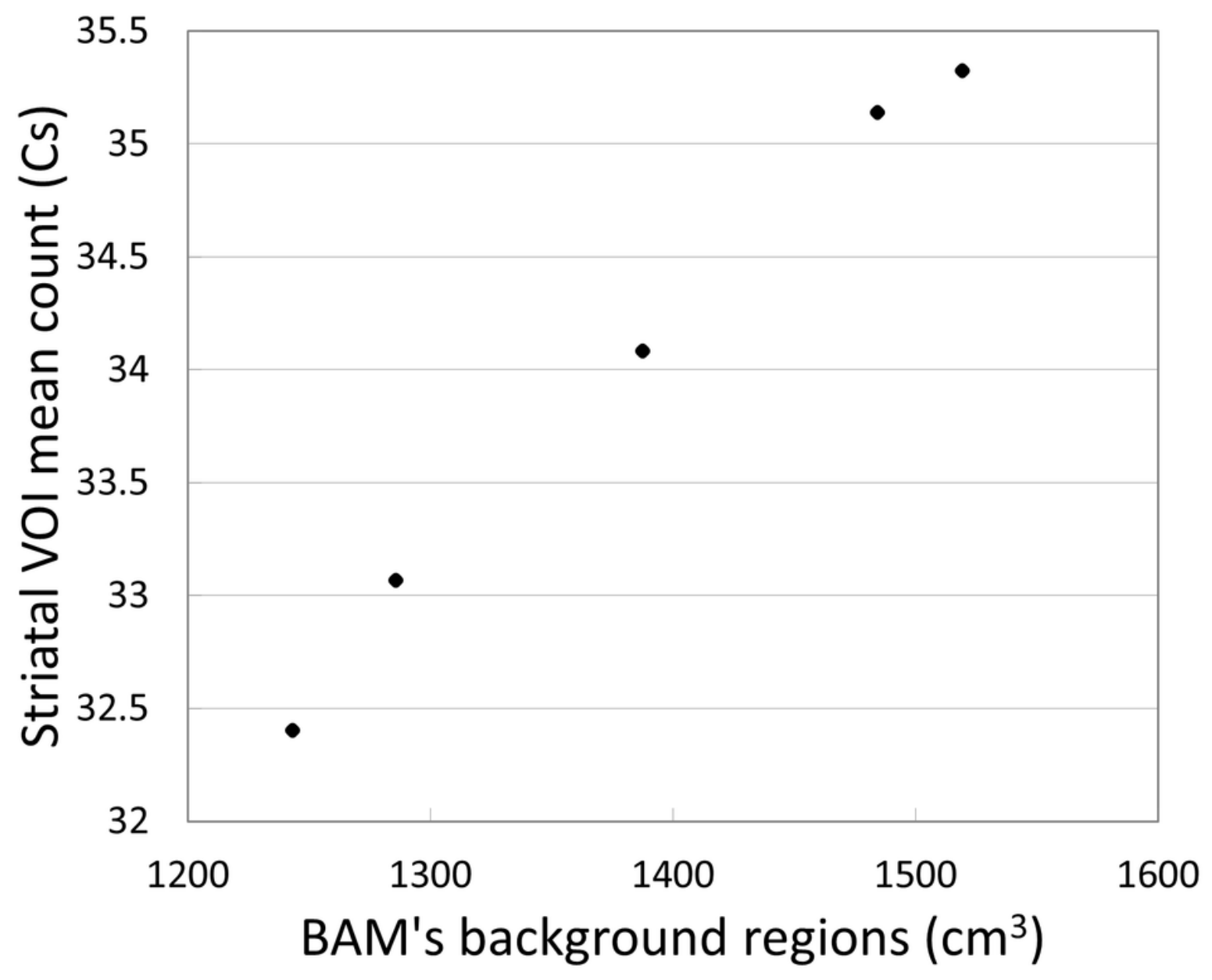

Figure 3

The relationship between $\mathrm{Cs}$ and the volume of the BAM background regions is displayed. Cs linearly increased with the volume of the BAM background regions increased. 


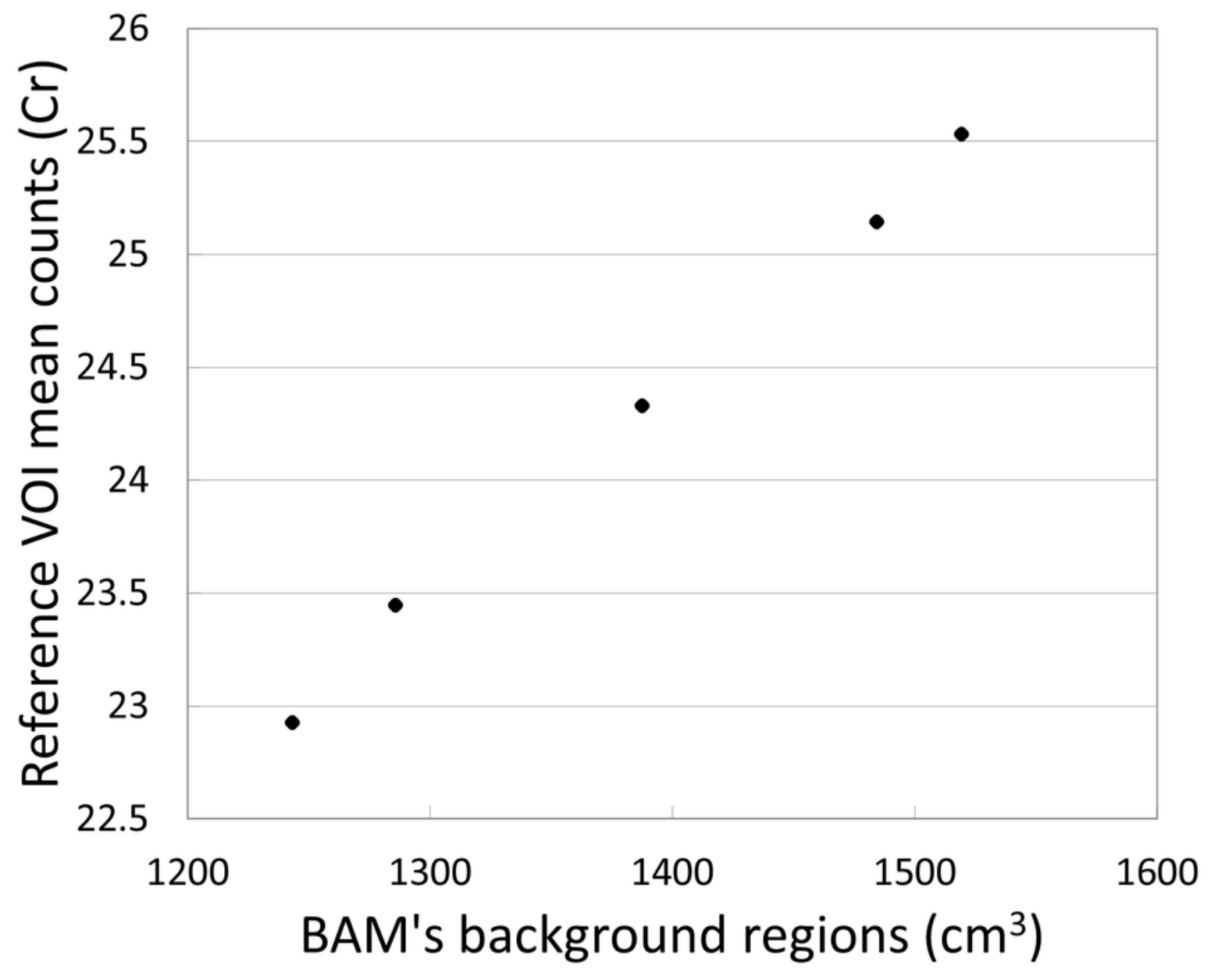

Figure 4

The relationship between $\mathrm{Cr}$ and the volume of the BAM background regions is displayed. $\mathrm{Cr}$ linearly increased with the volume of the BAM background regions increased. 


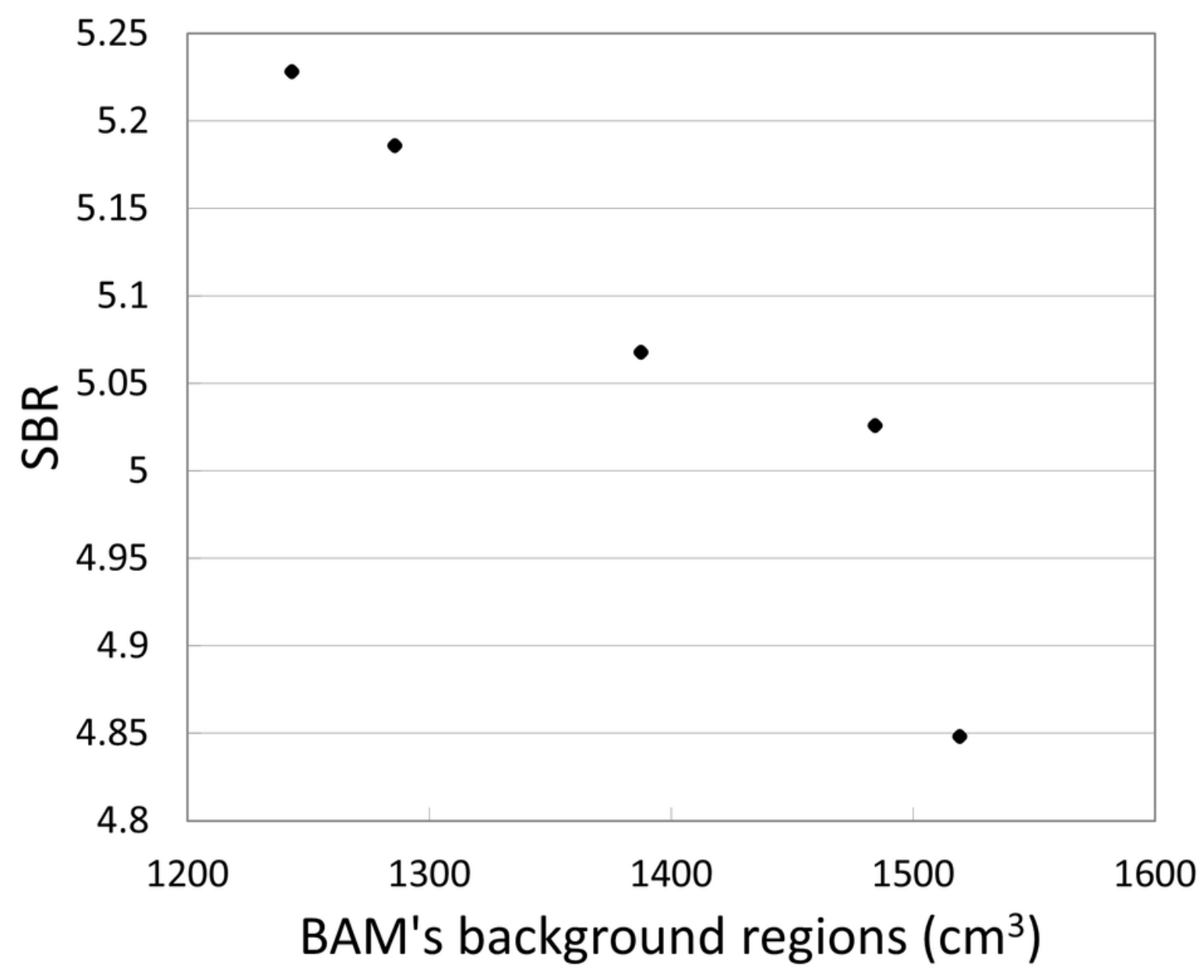

Figure 5

The relationship between SBR and the volume of the BAM background regions is displayed. SBR decreased as the volume of the BAM background regions increased. 